\title{
COMPORTAMENTO DO JATO DE AÇO E ANÁLISE DAS FLUTUAÇÕES SUPERFICIAIS EM UM MOLDE DE BEAM BLANK*
}

\section{Resumo}

Johne Jesus Mol Peixoto ${ }^{1}$ Weslei Viana Gabrie/ ${ }^{1}$ Ciro Azevedo Silva ${ }^{2}$ Leticia Queiroz Ribeiro ${ }^{2}$ Carlos Antonio da Silva ${ }^{3}$ Itavahn Alves da Silva ${ }^{3}$ Varadarajan Seshadrit

A complexidade do formato do molde de beam blank aumenta a importância de se compreender o comportamento do fluxo no interior do mesmo. Neste estudo verificou-se a influência da profundidade de imersão e da velocidade de lingotamento sobre as características de fluxo em um molde de beam blank alimentado por duas válvulas tubulares. Os resultados das simulações matemáticas foram coerentes com os resultados do modelamento físico. A velocidade de espalhamento do jato na região do flange é maior que na alma e este fator pode acarretar na solidificação irregular da pele ao longo da seção transversal. A profundidade de penetração do jato variou entre $66 \mathrm{~cm}$ e $77 \mathrm{~cm}$. A intensidade média de flutuação do menisco foi de $0,22 \mathrm{~mm}$, sendo a flutuação máxima encontrada de $0,85 \mathrm{~mm}$. Com base nos resultados obtidos, os parâmetros de operação sugeridos são: profundidade de imersão igual a $75 \mathrm{~mm}$ e velocidade de lingotamento máxima de $1 \mathrm{~m} / \mathrm{min}$.

Palavras-chave: Beam Blank; Fluxo; PIV; PLIF.

\section{BEHAVIOUR OF STEEL JET AND ANALYSIS OF SURFACE FLUCTUATIONS IN A BEAM BLANK MOLD}

\section{Abstract}

The complexity of the beam blank mold geometry increases the importance of assessing the flow behavior inside the mold. This work is related to the influence of the immersion depth and the casting speed on the flow characteristics in a beam blank mold fed with two tubular nozzles. There was found a good agreement between the results obtained from physical modeling and CFD modeling. The liquid jet spreads itself faster at the flange region than at the web and this factor can lead to an non uniform shell thickness along the cross section. The jet penetration depth ranged between $66 \mathrm{~cm}$ and $77 \mathrm{~cm}$. The average intensity of meniscus fluctuation was $0.22 \mathrm{~mm}$, and the maximum fluctuation was $0.85 \mathrm{~mm}$. Based on modeling results the suggested operating parameters are: SEN immersion of $75 \mathrm{~mm}$ and maximum casting speed of $1 \mathrm{~m} / \mathrm{min}$.

Keywords: Beam Blank; Fluid Flow; PIV; PLIF.

1 Engenheiro Metalurgista, Mestrando em Engenharia de Materiais, Redemat, Universidade Federal de Ouro Preto, Ouro Preto, Minas Gerais, Brasil.

2 Graduando em Metalurgia, Escola de Minas, Ouro Preto, Minas Gerais, Brasil.

3 Engenheiro Metalúrgico, Ph.D., Professor, Universidade Federal de Ouro Preto, Escola de Minas, Ouro Preto, Minas Gerais, Brasil.

4 Engenheiro Metalurgista, Dr. Ing., Professor Emérito, Universidade Federal de Minas Gerais (UFMG), Belo Horizonte, Minas Gerais, Brasil. 


\section{INTRODUÇÃO}

Yoon [1] aponta a alta eficiência metalúrgica do processo de lingotamento contínuo, em virtude dos muitos avanços tecnológicos obtidos. Um desses notáveis avanços tecnológicos é sem dúvida o lingotamento de pré-formas, tecnologia que consiste em obter o produto com geometria mais próxima da forma final de aplicação, reduzindo custos operacionais e aumentando a produtividade.

Onishi et al. [2], destacam que devido ao formato do produto, o lingotamento contínuo de beam blank difere consideravelmente do lingotamento de placas e tarugos quanto às tensões internas e mecanismos de solidificação. Chen et al. [3] e Lee et al. [4], entre outros, ressaltam que a transferência de calor entre o veio e as paredes do molde, padrão do campo de velocidades (particularmente na região do menisco), padrão de oscilação do molde e fenômenos de segregação, durante o regime de solidificação, têm grande influência na qualidade interna e superficial do produto. Neste sentido, Kim et al. [5], Lee et al. [4] e Hibeller et al. [6] detectaram a formação de um "gap" de ar na ponta do flange na etapa inicial da solidificação, o qual prejudica o processo de extração de calor, decrescendo a velocidade de solidificação. De igual maneira, o impacto do jato de aço líquido retarda o crescimento da pele na região do filete.

$\mathrm{Na}$ zona de refrigeração secundária do processo de lingotamento contínuo de beam blank, Zhao et al. [7] verificaram que trincas longitudinais na região do filete são geradas em decorrência da grande diferença de temperatura entre o filete e outras regiões na superfície do veio. Estes pesquisadores propuseram uma melhoria neste sistema de refrigeração, de modo a uniformizar a temperatura superficial e otimizar o consumo de água.

A geometria do bocal da(s) válvulas submersa(s) influencia o campo de velocidades e a dissipação de calor na região do molde. Neste contexto, Xu e Zhu [8] compararam o uso de válvulas tubulares simples e válvulas dotadas de três furos laterais na saída, dois apontados para o flange e um para a região central da alma. Os resultados mostraram que para válvulas tubulares, o perfil de temperatura é tal que a pele solidificada é mais fina na região do filete e mais espessa na alma; enquanto que para as válvulas com três furos laterais, ocorreu uma maior oscilação do menisco, porém o encontro dos jatos na região central da alma cria uma zona de alta temperatura, o que gera uma pele mais fina, e, por isso, propensa a rupturas.

Neste trabalho, aplicaram-se métodos de modelagem física e matemática para evidenciar os efeitos de velocidade de lingotamento e profundidade de imersão das válvulas submersas sobre o comportamento do fluxo no molde de lingotamento contínuo de beam blank.

\section{MATERIAIS E MÉTODOS}

\subsection{Modelamento Físico}

Os experimentos de modelagem física foram realizados em uma réplica em acrílico de um molde de lingotamento contínuo de beam blank, na escala 1:1, com dimensões $499 \mathrm{~mm} \times 415 \mathrm{~mm} \times 125 \mathrm{~mm}$ e altura de $1,5 \mathrm{~m}$. Nos testes foram empregadas válvulas submersas do tipo tubular com diâmetro interno de $34,6 \mathrm{~mm}$. As vazões de abastecimento do molde foram 100L/mim, $125 \mathrm{~L} / \mathrm{mim}$ e $150 \mathrm{~L} / \mathrm{mim}$, as quais correspondem às velocidades de lingotamento de $0,78 \mathrm{~m} / \mathrm{mim}, 0,98 \mathrm{~m} / \mathrm{mim}$ e $1,2 \mathrm{~m} / \mathrm{mim}$ na máquina industrial, respectivamente. As profundidades de imersão das 
válvulas submersas foram de $100 \mathrm{~mm}, 75 \mathrm{~mm}$ e $50 \mathrm{~mm}$. O padrão do fluxo foi analisado ao longo dos planos AA e BB, possibilitando uma descrição na região da alma e do flange (vide Figura 1.b e 1.d). A flutuação do menisco foi captada com o auxílio de um sensor ultrassônico de medição de distância (modelo Sick UM30211118) posicionado no ponto $\mathrm{P} 1$ e no ponto $\mathrm{P} 2$, para analisar a flutuação na região da alma e do filete, conforme a Figura 1.d. Foram realizados dois testes para cada uma das combinações de vazão e imersão das válvulas submersas, em cada ponto de análise. A posição do menisco foi medida em função do tempo, ao longo de $60 \mathrm{~s}$, e os dados foram utilizados para calcular a intensidade de flutuação média e máxima para cada teste.

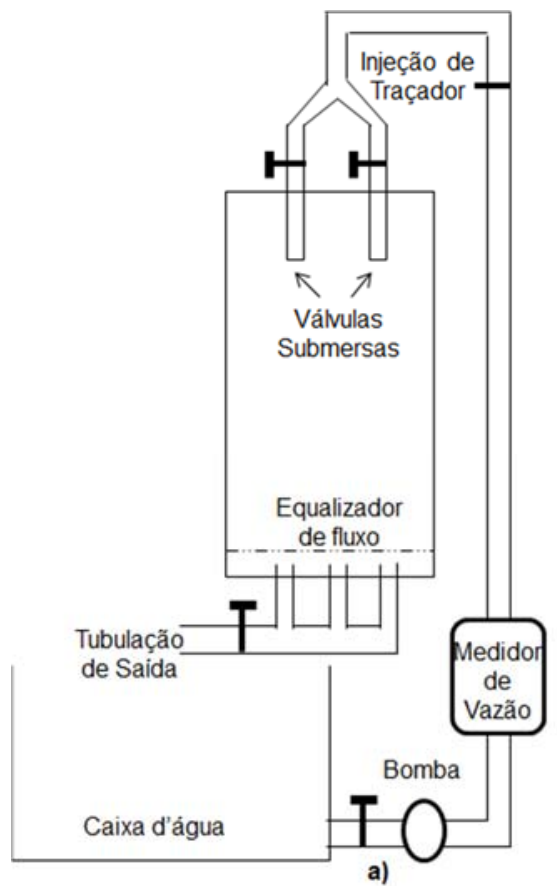

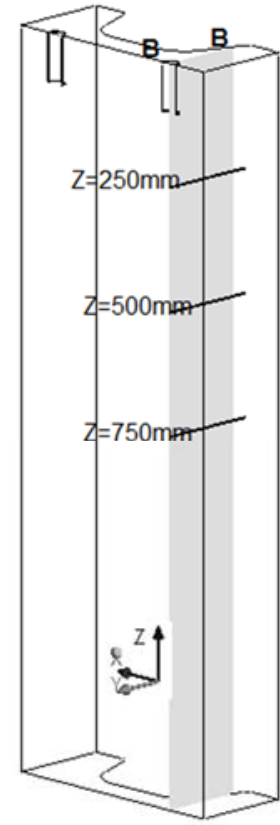

b)

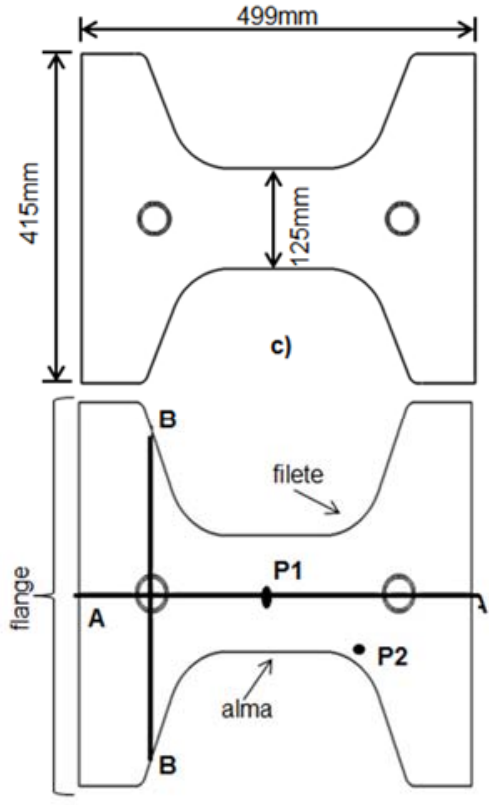

d)

Figura 1. a) Esquema do circuito utilizado nas simulações físicas; b) vista em perspectiva do molde de beam blank cortado pelo plano de simetria $A A ; c)$ dimensões do beam blank; d) seção transversal do molde de beam blank com suas regiões, planos de análise AA e BB e pontos de medição da flutuação do menisco P1 e P2.

Utilizou-se a técnica de injeção de tinta para caracterizar o comportamento do jato de aço descarregado no molde. Este método consiste em injetar o traçador na tubulação de entrada da água e filmar o caminho percorrido no interior do molde até seu total espalhamento, possibilitando a visualização do fluxo e a profundidade de penetração do jato.

A técnica PLIF (Planar Laser-Induced Fluorescence) foi utilizada (software Dynamics, montagem PIV da DANTEC®) para uma análise qualitativa do fluxo no interior do molde. Esta técnica faz uso da propriedade de fluorescência de alguma substância, denominada "traçador", para revelar o desenvolvimento e percurso do fluxo analisado. Como esquematizado na Figura 2, a técnica consiste em iluminar uma determinada região do molde com um plano de laser pulsante (comprimento de onda 532nm), que ao incidir sobre as moléculas do traçador (no caso a Rodamina 6B) as excita, fazendo com que a mesma emita uma radiação (comprimento de onda entre 540nm e 640nm). As imagens são capturadas por uma câmera CCD equipada com um filtro de luz permeável ao comprimento de onda $570 \mathrm{~nm}$, tornando possível o mapeamento do fluxo devido à correlação existente entre intensidade da radiação 
emitida e concentração do traçador, previamente estabelecida através de uma curva de calibração.

Para analisar quantitativamente o fluxo, por meio de simulações físicas, foi utilizada a técnica PIV (Particle Image Velocimetry). Este método consiste na dispersão de partículas com densidade igual à da água e incidência de um feixe de laser em pulsos, o que permite mapear o caminho percorrido no interior do fluido através de um processo de interpolação. Uma câmera CCD captura uma sequência de imagens, que são processadas através de um software específico (Dynamics da DANTEC $\circledast$ ), fornecendo os valores de velocidades, vorticidade e flutuações, dentre outros parâmetros do fluxo.

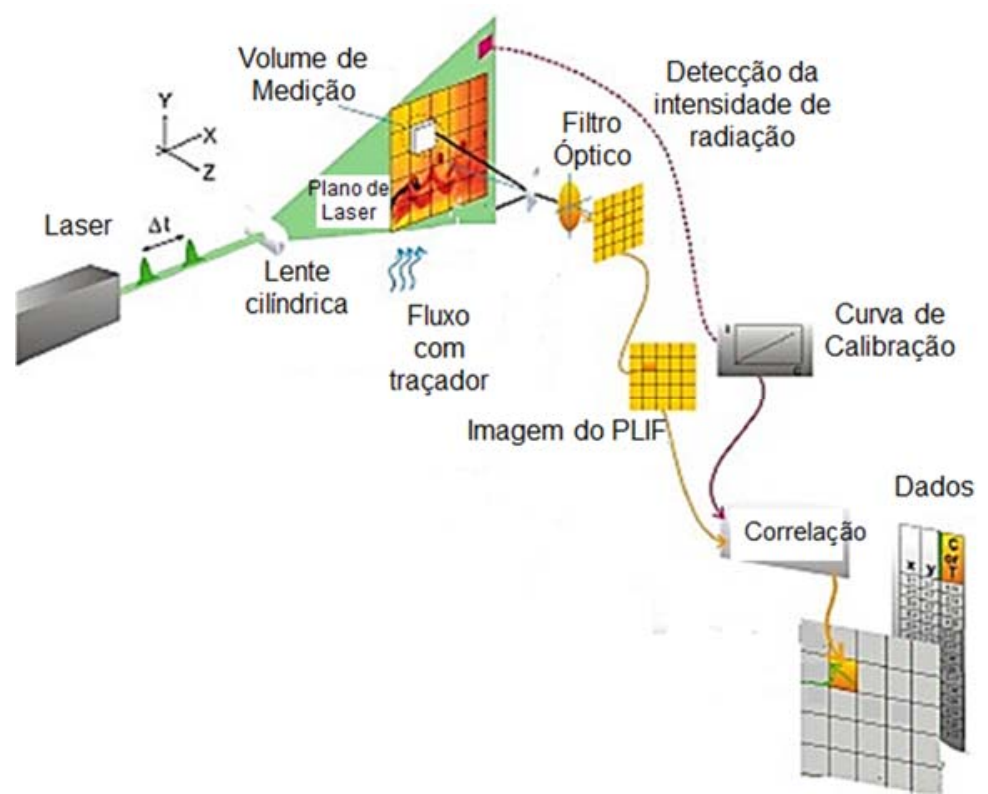

Figura 2. Esquema do funcionamento de um aparelho Planar LIF, de acordo com a DANTEC@.

\subsection{Modelamento Matemático}

As simulações computacionais foram realizadas com o software Ansys ${ }^{\circledR}$ CFX 15.0 versão acadêmica, em regime permanente, considerando a água um fluido Newtoniano incompressível, com temperatura constante e igual a $25^{\circ} \mathrm{C}$. O modelo de turbulência empregado foi o k- $\varepsilon$, no qual se resolvem as equações da continuidade, de Navier-Stokes e da viscosidade efetiva, além das equações auxiliares para determinação de $k$ (energia cinética de turbulência) e $\varepsilon$ (taxa de dissipação da energia cinética de turbulência).

Condições de contorno aplicáveis ao problema:

- Condição de não deslizamento nas paredes do molde e das válvulas;

- Condição de livre deslizamento no menisco;

- Condição de simetria, o que implica que o cálculo em questão abrange apenas metade de toda a geometria, e nessas regiões as componentes de velocidade normais à fronteira são iguais a zero.

- Vazão mássica na entrada: 0,833kg/s, $1,042 \mathrm{~kg} / \mathrm{s}$ e $1,25 \mathrm{~kg} / \mathrm{s}$ para as velocidades de lingotamento equivalentes a $0,78 \mathrm{~m} / \mathrm{min}, 0,98 \mathrm{~m} / \mathrm{min}$ e $1,2 \mathrm{~m} / \mathrm{min}$, respectivamente.

Para avaliar o comportamento do traçador foi conduzida uma simulação matemática em regime transiente com tempo total de 15s. Neste caso, o resultado da simulação matemática em regime permanente nas mesmas condições de vazão e imersão da 
SEN (Submerged Entry Nozzle) foi utilizado como condição inicial, para garantir que o traçador foi injetado no molde com fluxo completamente desenvolvido.

\section{RESULTADOS E DISCUSSÃO}

\subsection{Análise do Perfil de Velocidades}

O perfil de velocidades foi determinado em três partes do plano BB através da técnica PIV, analisando uma profundidade total de $800 \mathrm{~mm}$. Na Figura 3 são comparados os resultados deste teste e da simulação computacional. Observou-se uma boa coerência em relação à estrutura do jato e localização dos vórtices. Da mesma forma, os gráficos da Figura 4 relacionam a distância y do centro da válvula submersa com a componente vertical de velocidade, analisadas numa linha horizontal que corta o plano BB, nas distâncias de $250 \mathrm{~mm}, 500 \mathrm{~mm}$ e $750 \mathrm{~mm}$, a partir do menisco (Figura 1.b). Comparando os resultados obtidos via simulações físicas (PIV) com os obtidos via simulações computacionais (CFD), observou-se mesma intensidade nas velocidades, porém, as curvas geradas pela simulação computacional mostram-se mais suaves e, com isto, o jato descarregado no molde mostrou-se mais aberto que aquele obtido via a técnica PIV.

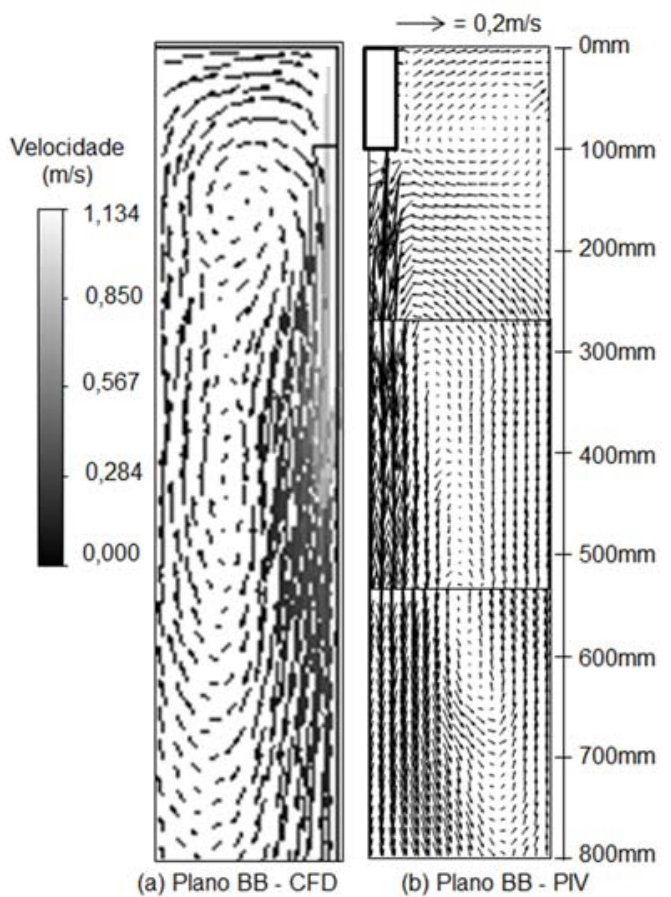

Figura 3. Perfil de velocidades para vazão de 100L/min e imersão da SEN 100mm: a) seção BBCFD e b) seção BB - PIV. 

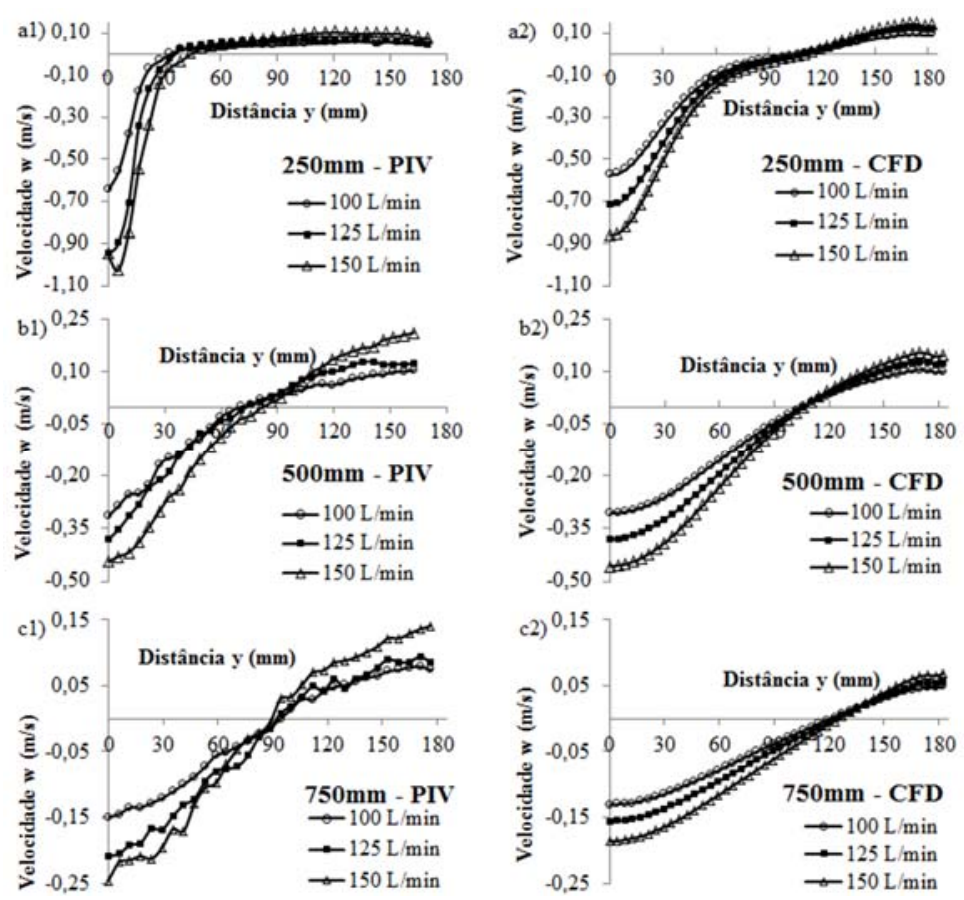

Figura 4. Gráficos de velocidade em função da distância y do centro da válvula submersa até a ponta do flange, obtidos: pela técnica PIV a uma distância do menisco de a1) $250 \mathrm{~mm}$, b1) $500 \mathrm{~mm}$ e c1) $750 \mathrm{~mm}$; por simulação numérica no CFD a uma distância do menisco de a2) $250 \mathrm{~mm}$, b2) $500 \mathrm{~mm}$ e c2) $750 \mathrm{~mm}$.

A Figura 5 mostra que a mudança na profundidade de imersão das válvulas de $50 \mathrm{~mm}$ para $75 \mathrm{~mm}$ não altera significativamente o padrão de fluxo. A posição dos vórtices e a distribuição no campo vetorial de velocidades mostram-se praticamente as mesmas para ambas as condições. Nestas imersões, existem seis vórtices no total, dois na alma e quatro nos flanges, assim como foi descrito em trabalho anterior (Peixoto et al. [9]) para a imersão de $100 \mathrm{~mm}$. Este padrão de fluxo também foi observado nos trabalhos de Lee et al. [4] e Chen et al. [10].

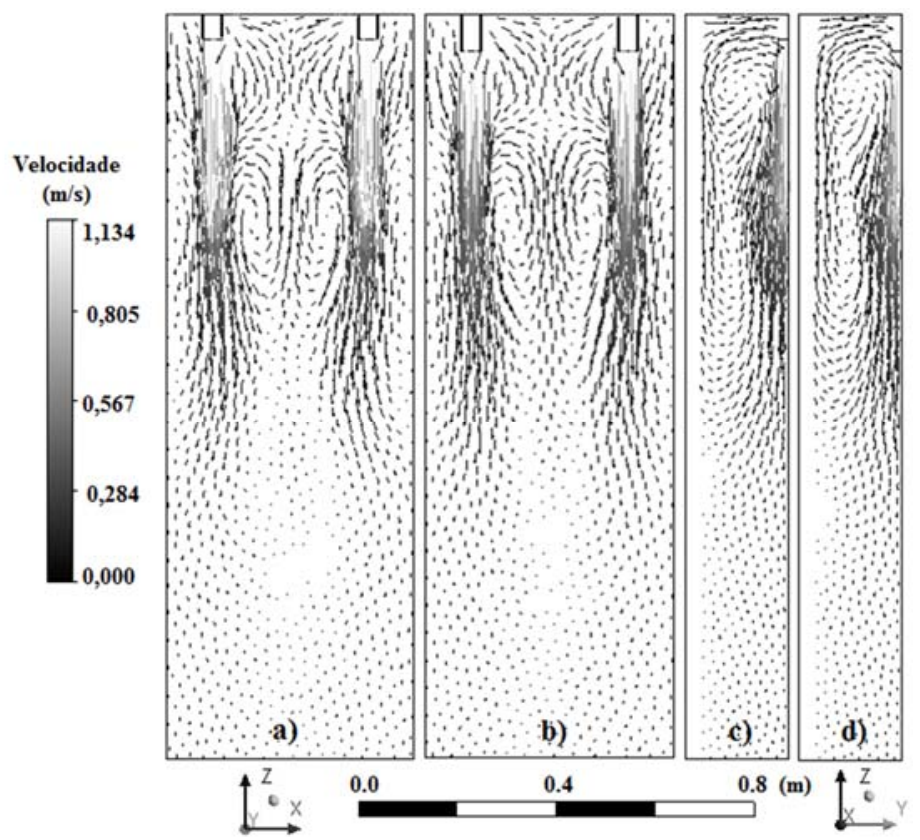

Figura 5. Perfil de velocidades obtido por simulação matemática para vazão de 125L/min: Plano AA a) imersão $50 \mathrm{~mm}$ e b) imersão $75 \mathrm{~mm}$; plano BB c) imersão $50 \mathrm{~mm}$ e d) imersão $75 \mathrm{~mm}$. 


\subsection{Caracterização do Jato}

A Figura 6 mostra que, sob tais condições de vazão e imersão, a profundidade dos jatos descarregados no molde foi de aproximadamente $0,8 \mathrm{~m}$, considerando o tempo $\mathrm{t}=2 \mathrm{~s}$, pois neste instante aparentemente tem-se apenas a ação da força de arraste proveniente dos jatos que saem das válvulas sobre o traçador (tinta), e para tempos superiores há um maior espalhamento da tinta. Na simulação computacional transiente utilizando o traçador, nas mesmas condições de imersão e vazão, tem-se uma profundidade de penetração do jato que sai da válvula submersa da ordem de $0,75 \mathrm{~m}$, para um tempo $\mathrm{t}=2 \mathrm{~s}$ (Figura 7.c). Tanto os resultados do teste físico quanto do modelamento matemático mostraram que 0 traçador se espalha mais rapidamente para os flanges e lentamente pela alma. Notou-se que para um tempo de 10s o traçador ainda não cobre a região da alma na parte superior do menisco, evidenciando que a renovação de fluido nesta região é lenta. Isto implica em um menor aporte térmico, pois o aço já chega "frio" nesta região e, consequentemente, a pele será mais espessa na região da alma. É importante ressaltar ainda que o impacto do fluxo de aço sobre a pele sólida nas regiões do filete e do centro do flange retarda o crescimento da pele nessas regiões. Este efeito na região central do flange, também foi observado por Xu e Zhu [8], que o denominaram "washing effect".

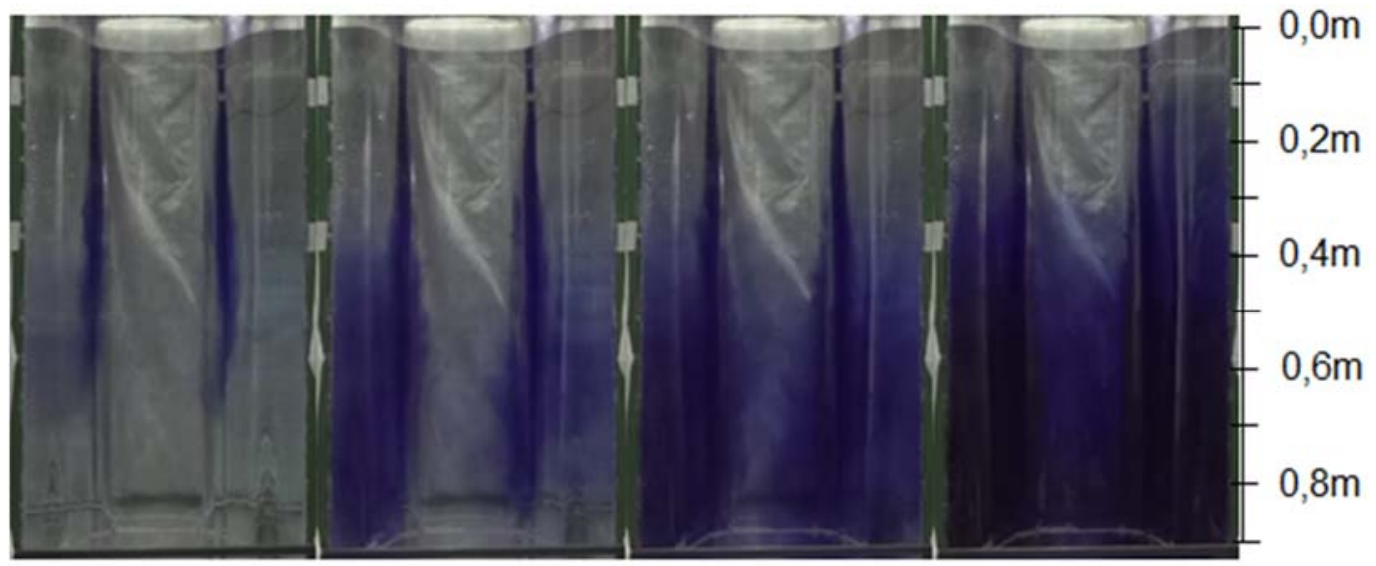
(a) $1 \mathrm{~s}$
(b) $2 \mathrm{~s}$
(c) $4 \mathrm{~s}$
(d) $10 \mathrm{~s}$

Figura 6. Espalhamento de traçador (tinta) inserido na tubulação de alimentação do molde $-a) t=1 s$; b) $\mathrm{t}=2 \mathrm{~s} ; \mathrm{c}$ ) $\mathrm{t}=4 \mathrm{~s}$ e d) $\mathrm{t}=10 \mathrm{~s}$. Vazão $125 \mathrm{~L} /$ mine imersão $100 \mathrm{~mm}$.

Os valores da profundidade de penetração do jato, obtidos por simulação matemática, estão apresentados na Tabela 1. O aumento da vazão e da imersão da SEN implicou em aumento na profundidade do jato. Chen et al. [10] encontraram a profundidade de impacto superior a $1 \mathrm{~m}$ para profundidades de imersões da SEN variando de $25 \mathrm{~mm}$ a $125 \mathrm{~mm}$; enquanto que, Zhang et al. [11] obtiveram por modelamento físico valores entre $50 \mathrm{~cm}$ e $60 \mathrm{~cm}$, para profundidades de imersão da SEN entre $50 \mathrm{~mm}$ e $110 \mathrm{~mm}$.

Tabela 1. Profundidade de penetração do jato em função da vazão e da imersão da SEN.

\begin{tabular}{|c|c|c|c|c|c|c|c|c|c|}
\hline Imersão da SEN & \multicolumn{3}{|c|}{$50 \mathrm{~mm}$} & \multicolumn{3}{c|}{$\mathbf{7 5 m m}$} & \multicolumn{3}{c|}{ 100mm* } \\
\hline Vazão (L/min) & 100 & 125 & 150 & 100 & 125 & 150 & 100 & 125 & 150 \\
\hline $\begin{array}{c}\text { Profundidade do jato } \\
\text { (cm) }\end{array}$ & 66,3 & 70,8 & 75,3 & 69,7 & 75,3 & 77,5 & 74,0 & 75,5 & 77,3 \\
\hline
\end{tabular}




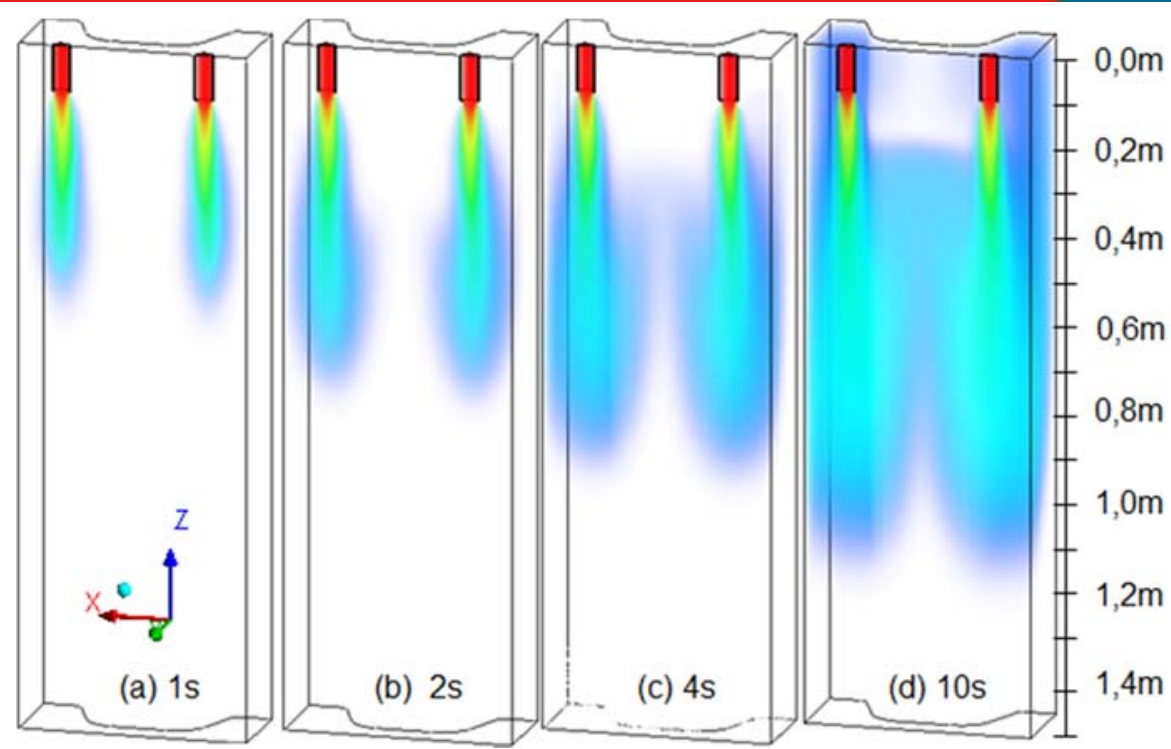

Figura 7. Espalhamento de traçador obtido por simulação matemática em regime transiente $-a) t=1 s$; b) $\mathrm{t}=2 \mathrm{~s}$; c) $\mathrm{t}=4 \mathrm{~s}$ e d) $\mathrm{t}=10 \mathrm{~s}$. Vazão $125 \mathrm{~L} / \mathrm{min}$ e imersão $100 \mathrm{~mm}$.

A Figura 8 apresenta uma comparação do espalhamento de traçador (Rodamina 6B) para as profundidades de imersões de $75 \mathrm{~mm}$ e $100 \mathrm{~mm}$. Deve-se destacar o caráter transiente do jato, que para $t=1 \mathrm{~s}$ apresentou pequenos desvios em relação ao seu eixo de simetria vertical. Após o espalhamento do jato, a força inercial, responsável pelo desenvolvimento na direção vertical, deixa de ser a predominante no sistema e o mesmo se espalha por convecção, retornando em direção ao menisco, seguindo o movimento dos vórtices da região do flange. Nota-se que a redução da profundidade imersão da válvula submersa implicou em maior espalhamento do jato, sendo que para $\mathrm{t}=15 \mathrm{~s}$ a região superior da ponta do flange está preenchida de traçador para a imersão de $75 \mathrm{~mm}$ (Figura 8.b) e que o mesmo não ocorreu para a profundidade de imersão da válvula submersa de 100mm (Figura 8.a).
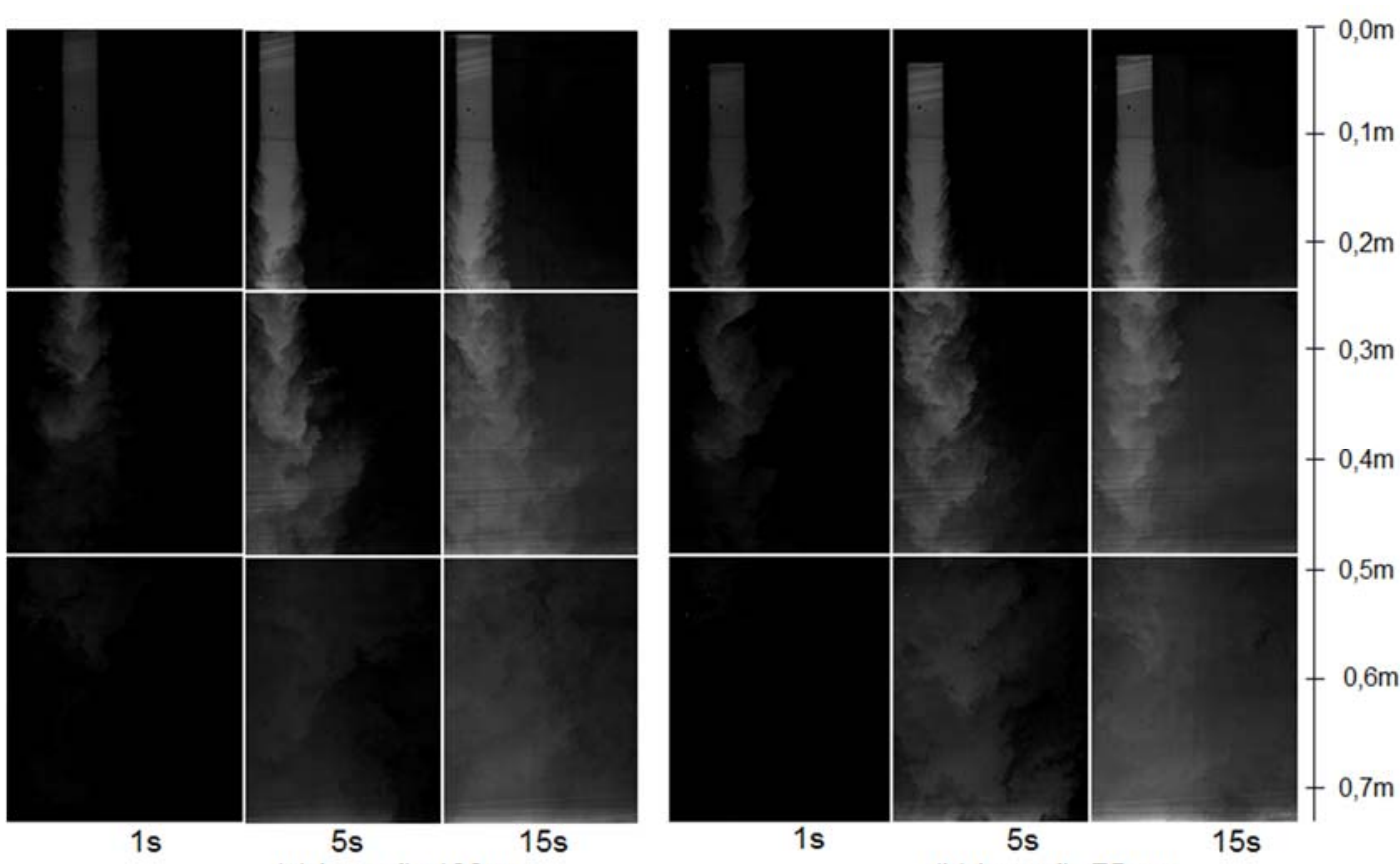

(a) Imersão $100 \mathrm{~mm}$

(b) Imersão $75 \mathrm{~mm}$

Figura 8. Espalhamento de traçador (Rodamina 6B) obtido via técnica PLIF no plano BB para vazão de 125L/min: a) Imersão 100mm e b) Imersão 75mm. 


\subsection{Avaliação da flutuação do Menisco}

A Figura 9 apresenta os resultados da análise da flutuação no menisco. A intensidade média de flutuação foi da ordem de $0,22 \mathrm{~mm}$ e a intensidade máxima foi ligeiramente maior na região da alma $(0,85 \mathrm{~mm})$ que na região do filete $(0,75 \mathrm{~mm})$. A redução da profundidade de imersão da SEN não resultou em variação significativa da intensidade de flutuação do menisco. Estes dados mostraram que utilizando as válvulas tubulares simples, a superfície livre do menisco praticamente não oscila, devido à alta profundidade de penetração do jato, o que é prejudicial para o processo de fusão do pó fluxante e flotação de inclusões.

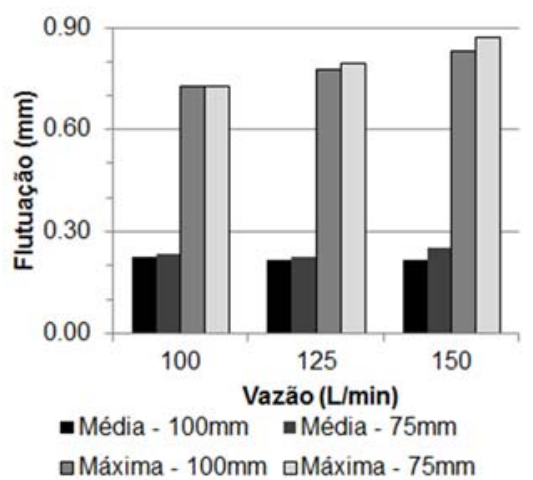

(a) P1 - Alma

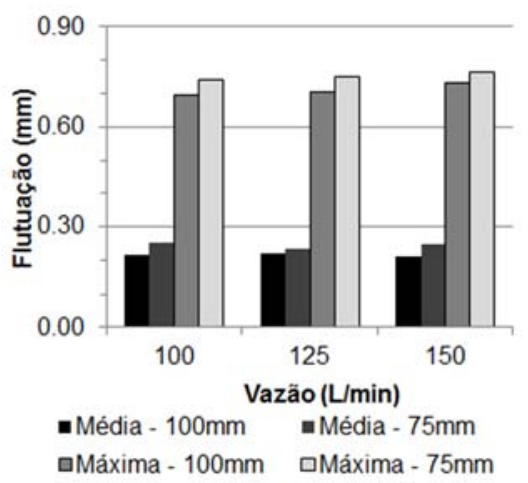

(b) P2 - Filete

Figura 9. Gráfico da amplitude de oscilação do menisco para diferentes vazões e imersões: a) Alma P1; b) Filete - P2.

Houve substancial coerência entre os resultados obtidos, com aqueles disponíveis na literatura acerca da flutuação do menisco. Por simulação matemática, Xu e Zhu [8] observaram que para válvulas tubulares, o nível de flutuação do menisco mostrou-se menor que $1 \mathrm{~mm}$; enquanto que Zhang et al. [11], via modelamento físico, obtiveram uma flutuação média da ordem de $0,2 \mathrm{~mm}$ para SEN de $40 \mathrm{~mm}$ de diâmetro interno e $70 \mathrm{~mm}$ de profundidade de imersão. Já Chen et al. [10], obtiveram, por simulação matemática, uma flutuação de oscilação da ordem de $0,8 \mathrm{~mm}$ na região da alma e $2,2 \mathrm{~mm}$ na do flange, para imersão de $75 \mathrm{~mm}$.

A partir dos resultados anteriores, sugere-se uma profundidade de imersão das válvulas submersas próxima de $75 \mathrm{~mm}$ e velocidade de lingotamento máxima de $1 \mathrm{~m} / \mathrm{min}$.

\section{CONCLUSÃO}

Os resultados das simulações matemáticas mostraram-se coerentes com os resultados do modelamento físico. Utilizando-se duas válvulas tubulares com diâmetro interno de $34,6 \mathrm{~mm}$, observou-se que:

- O traçador se espalha mais rapidamente para a ponta dos flanges e lentamente pela alma;

- A profundidade de penetração do jato variou entre $66 \mathrm{~cm}$ e $77 \mathrm{~cm}$;

- A intensidade média de flutuação do menisco foi da ordem de $0,22 \mathrm{~mm}$ e a intensidade máxima de flutuação foi $0,85 \mathrm{~mm}$. Este parâmetro mostrou-se não ser afetado significativamente pela variação da velocidade de lingotamento e da profundidade de imersão da SEM. 


\section{Agradecimentos}

Os autores gostariam de agradecer ao Conselho Nacional do Desenvolvimento Científico e Tecnológico (CNPq), à Coordenação de Aperfeiçoamento de Pessoal de Nível Superior (CAPES), Fundação Gorceix e à FAPEMIG pelo apoio.

\section{REFERÊNCIAS}

1 Yoon, J-K. Applications of Numerical Simulation to Continuous Casting Technology. ISIJ International. 2008;48(7):879-884.

2 Onishi M, Mizota H, Ueda T, Yao M, Shinjo Y, Fujimura T. Continuous Casting of Beam Blanks. Kawasaki Steel Technical Report. 1981; ${ }^{\circ} 3: 13-25$.

3 Chen W, Zhang YZ, Zhang CJ, Zhu LG, Wang BX, Lu WG, Ma JH. ACTA Metallurgica Sinica (English Letters). 2007;20(4):241-250.

4 Lee J-E, Yeo T-J, Oh KH, Yoon J-K, Yoon U-S. Prediction of Cracks in Continuously Cast Steel Beam Blank through Fully Coupled Analysis of Fluid Flow, Heat Transfer, and Deformation Behavior of a Solidifying Shell. Metallurgical and Materials Transactions. 2000;31(A):225-237.

$5 \quad \mathrm{Kim} \mathrm{K}$, Han HN, Yeo T, Lee Y, Oh KH, Lee DN. Analysis of surface and internal cracks in continuously cast beam blank. Ironmaking and Steelmaking. 1997;24(3):249-256.

6 Hibbeler LC, Xu K, Thomas B.G, Koric S, Spangler C. Thermomechanical Modeling of beam blank Casting. Iron \& steel technology. 2009;6(7):60-73.

7 Zhao Y, Chen DF, Long MJ, Shen JL, Qin RS. Two-dimensional heat transfer model for secondary cooling of continuously cast beam blanks. Ironmaking and Steelmaking. 2014;41(5):377-386.

8 Xu M, Zhu M. Transport Phenomena in a Beam-Blank Continuous Casting Mold with Two Types of Submerged Entry Nozzle. ISIJ International. 2015;55(4):791-798.

9 Peixoto JJM, Gabriel WV, Ribeiro LQ, Souza SS, Silva CA, Silva IA, Seshadri V. Análise do Fluxo em Molde de Lingotamento Contínuo de Beam Blank: Simulação Computacional e Física. $46^{\circ}$ Seminário de Aciaria-Internacional ABM Week. 2015;1-10.

10 Chen W, Zhang Y-Z, Zhu L-G, Zhang C-J, Chen Y, Wang B-X, Wang C. Threedimensional FEM study of fluid flow in mould for beam blank continuous casting: influence of straight through conduit type SEN. Ironmaking and Steelmaking. 2012; 39(8):551-559.

11 Zhang L, Chen D, Long M, Xie X, Zhang X, Ma Y. Hydraulic Simulations of Fluid Flow in Beam Blank Casting Mold with Double Nozzles. EPD Congress. 2014;375-384. 